\title{
Thermal approach to classifying sequentially written fiber Bragg gratings
}

\author{
Senta L. Scholl, ${ }^{1, *}$ (i) Alexander Jantzen, ${ }^{1}$ (i) Rex H. S. Bannerman, ${ }^{1}$ (i) Paul C. Gow, ${ }^{1}$ (i) \\ Devin H. Smith, ${ }^{1}$ James C. Gates, ${ }^{1}{ }^{10}$ Lewis J. Boyd, ${ }^{2}$ Peter G. R. Smith, ${ }^{1}$ and Christopher Holmes ${ }^{1}$ (1) \\ ${ }^{1}$ Optoelectronics Research Centre, University of Southampton, Southampton, SO17 1BJ, UK \\ ${ }^{2}$ Parker Aerospace, Parker Hannifin Corporation, 2510 The Quadrant, Bristol, BS32 4AQ, UK \\ *Corresponding author: S.Scholl@soton.ac.uk
}

Received 2 October 2018; revised 7 December 2018; accepted 11 December 2018; posted 12 December 2018 (Doc. ID 345891); published 31 January 2019

\begin{abstract}
We demonstrate thermal classification of sequentially written fiber Bragg gratings. This Letter presents a process to determine the type of fiber Bragg grating written in SMF28 and GF4A by introducing the gratings to thermal treatment. This technique can be applied to several approaches based on sequential writing, including the small spot direct ultraviolet writing technique. Four different types of gratings have been identified, which are dependent on the fiber type and fluence used during the writing process.
\end{abstract}

Published by The Optical Society under the terms of the Creative Commons Attribution 4.0 License. Further distribution of this work must maintain attribution to the author(s) and the published article's title, journal citation, and DOI.

https://doi.org/10.1364/OL.44.000703

Fiber Bragg gratings (FBGs) are widely used for harsh environmental sensing due to their thermal stability, small footprint, chemical inertness, and immunity to electromagnetic interference $[1,2]$. For these reasons, FBGs find their application in various sectors, for example, automotive, civil engineering, and aerospace [3]. The broad temperature range in aerospace environments is specifically challenging for sensor development, as they can range from $-55^{\circ} \mathrm{C}$ externally over $600^{\circ} \mathrm{C}$ near the brakes and over $1500^{\circ} \mathrm{C}$ in the engines [4,5]. Depending on the grating type, the FBGs can withstand temperatures up to and over $1000^{\circ} \mathrm{C}$. Hence, they form an ideal sensor platform for high-temperature environments [6,7]. To fabricate an optical sensor based on Bragg gratings, it is important to know the grating type, as this defines the operating range of the device.

There are eight different fiber Bragg grating types, summarized by Canning in Ref. [7]. For most FBGs, the fiber type (doped, hydrogenated, etc.), along with the laser source used to create those gratings, dictates the type of grating. However, some gratings, such as the regenerated gratings, require postprocessing and will be not discussed in this Letter.

As discussed by Canning, the physical features of these gratings are mainly dependent on the composition of the fiber and the writing process that induces the refractive index change in the fiber. The underlying mechanisms of the index change are not yet fully determined, but it is commonly accepted that defects cause a refractive index change in the material. The color center model explains the refractive index change by a photo-induced alteration of the absorption according to the Kramers-Kronig relations [8]. For higher doses of UV radiation, it is believed that densification has a greater impact on the refractive index change [9].

Oftentimes, gratings are simple to classify, as they show distinct features during the fabrication process. This is particularly apparent for germanosilicate fibers during the transition from Type I to Type In grating: during the initial exposure, the grating develops, and the central wavelength starts to red shift. With further exposure, the grating reflection becomes weaker and develops a blue shift. With the blue shift, the grating reflection gets stronger again [10]. This process is called roll-over and indicates the transition from Type I to Type In. The Type In gratings are known as negative index gratings. The fact that the spectral red or blue shift directly corresponds to the average refractive index gives a real-time indication of an index increase or decrease. It should be noted that this roll-over feature does not occur for hydrogen-loaded fibers, which typically transitions from Type IH to Type IHp.

Generally, spectral shifts during fabrication are used to classify different grating types. This method is established for writing techniques, such as the phase mask approach where the whole grating is exposed at once [11]. The work presented in this Letter classifies gratings written with the small spot direct ultraviolet writing (SSDUW) technique. Due to the writing technique being inherently different, a classification based on the spectral response cannot be pursued with the established technique, as the full grating response cannot be monitored during the writing process. This also applies to other sequential grating writing approaches. Therefore, the classification was achieved by thermal processing.

The SSDUW technique is a fabrication method, where the grating structure is formed using a small interference spot. Figure 1 displays the setup of the system. The beam of a frequency-doubled $\mathrm{cw}$ argon ion laser $(244 \mathrm{~nm})$ is split with a 


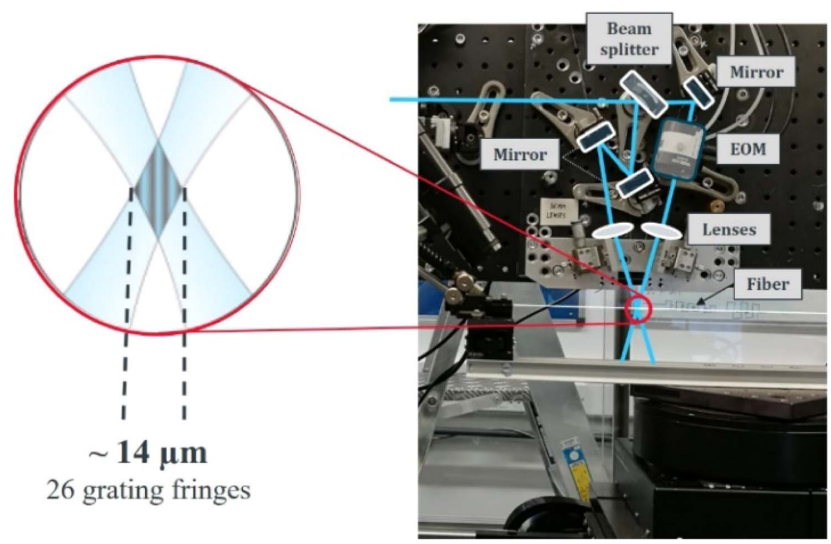

Fig. 1. Set up of the SSDUW system: the $244 \mathrm{~nm}$ laser beam is divided up into two arms that are recombined in the fiber core, creating an interference pattern.

beam splitter into two arms creating an interferometer. Both beams are recombined and focused in the fiber core, where they create an interference pattern. The fiber is stripped and held by fiber clamps on both ends with an applied tension of $0.5 \mathrm{~N}$. This setup is mounted on a translation stage that moves the fiber through the interference spot during the writing process. One arm of the interferometer is passed through an electrooptical modulator, which modulates the phase of the arm with an applied voltage and, hence, moves the interference fringes. When the fringes move with the same speed as the translation speed of the fiber, the constructive interference fringes expose the core and form a refractive index grating. This interferometric technique creates an interference pattern with a fixed period. However, by moving the grating fringes with a slightly slower (or slightly faster) speed than the fiber is translated through the beam, the grating period becomes larger (or smaller). This detuning allows the writing of gratings over a wide wavelength range [12]. Prior to writing, the interference spot is aligned at five positions along the length of the fiber. An algorithm creates an interpolation of these positions and corrects for the spot's spatial position during the writing process to ensure a continual alignment of the interference sport within the core.

Within this Letter, the crossing angle of the two UV laser beams was set to $26^{\circ}$, which results in an inherent interference period of $542 \mathrm{~nm}$. A $14 \mu \mathrm{m}$ spot will then include 26 grating fringes. Due to the small spot, greater control can be placed on the grating design compared to conventional UV writing techniques $[13,14]$.

Grating classification was performed for two different types of fiber: GF4A, a photosensitive cladding mode offset fiber, specifically developed by Nufern for FBG writing; and SMF28 from Corning, a fiber widely used in telecommunication. Gaussian apodized FBGs were written physically consecutively with an individual grating length of $5 \mathrm{~mm}$ in both fibers. An example of the spectral response of the different gratings in GF4A and SMF28 is displayed in Fig. 2. SMF28 was hydrogen loaded at 130 bars for two weeks prior to the writing process to enhance photosensitivity. Since GF4A has large inherent photosensitivity, it did not require hydrogen loading. The gratings were written at different fluences (energy per surface area) controlled by the translation speed of the fiber. The fluence
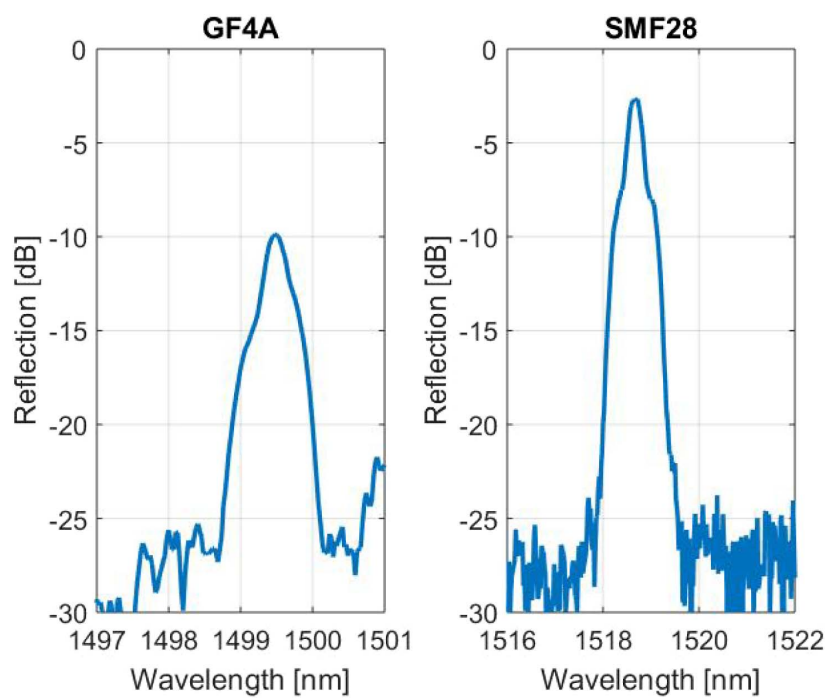

Fig. 2. Bragg reflection versus the wavelength for a grating written with $10 \mathrm{~kJ} / \mathrm{cm}^{2}$ in the photosensitive GF4A and with $7 \mathrm{~kJ} / \mathrm{cm}^{2}$ in hydrogen-loaded SMF28 collected at room temperature.

in the SSDUW technique is comparable to the exposure time in conventional fabrication methods where the whole grating is typically exposed at once. Therefore, the fluence determines the type of grating, along with the fiber type and its state of hydrogenation, as this defines the induced refractive index change. An overview of the different fluences that have been used to define the gratings and their reflections for both GF4A and SMF28 is represented in Fig. 3. These reflection spectra were measured at room temperature. The laser power was measured prior to each writing experiment to be approximately $53 \pm 1 \mathrm{~mW}$.

For the thermal treatment, the FBGs were inserted into a furnace (Seven Thermal Solutions CU2006), which was ramped up at $5^{\circ} \mathrm{C} / \mathrm{min}$ to $850^{\circ} \mathrm{C}$ for the GF4A fiber and to $750^{\circ} \mathrm{C}$ for the SMF28 fiber. The temperature was held at the peak temperature for 30 min after which it was ramped

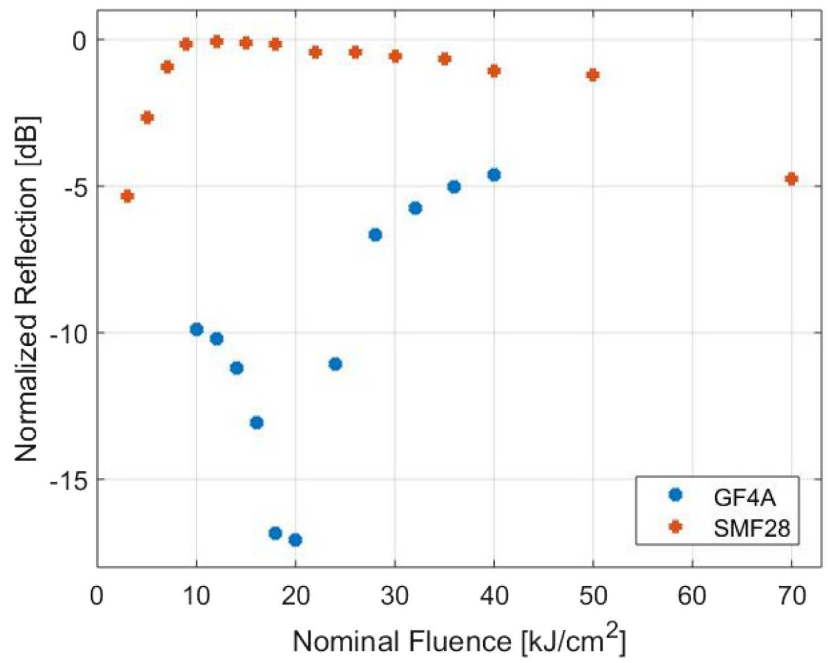

Fig. 3. Bragg reflection versus fluence for GF4A and hydrogenloaded SMF28 measured at room temperature. Notice that the GF4A has a minimum around $20 \mathrm{~kJ} / \mathrm{cm}^{2}$, indicating the characteristic roll-over from Type I to Type In. 


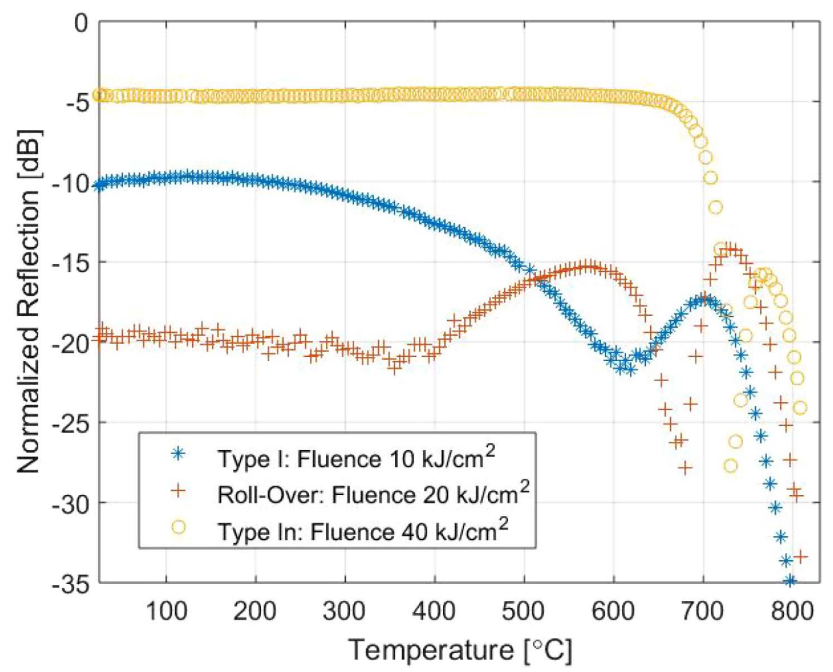

Fig. 4. Bragg reflection versus temperature for three distinct fluences in GF4A. The $40 \mathrm{~kJ} / \mathrm{cm}^{2}$ grating shows little thermal change up to $650^{\circ} \mathrm{C}$. The low fluence grating starts to show a decrease in reflection at $250^{\circ} \mathrm{C}$. A superposition of the two grating types causes the $20 \mathrm{~kJ} / \mathrm{cm}^{2}$ grating to gain reflection strength at $400^{\circ} \mathrm{C}$, indicating the annealing of the Type I component. All gratings show thermal regeneration.

down at $5^{\circ} \mathrm{C} / \mathrm{min}$. An argon gas purge was introduced $30 \mathrm{~min}$ prior to the ramp up in order to create an inert environment. The reflection spectrum was recorded during the thermal treatment using a broadband super luminescent source (Amonics ASLD-CWDM-5B-FA), covering the range from 1250 to $1650 \mathrm{~nm}$, and an optical spectrum analyzer (ANDO AQ6317B). A thermocouple was used to track the effective reference temperature inside the furnace.

The evolution of the reflection over the temperature for GF4A is presented in Fig. 4 for three different fluences. The lowest fluence grating $\left(10 \mathrm{~kJ} / \mathrm{cm}^{2}\right)$ shows a decay in reflection starting at $250^{\circ} \mathrm{C}$. The highest fluence grating $\left(40 \mathrm{~kJ} / \mathrm{cm}^{2}\right)$ demonstrates a constant reflection strength up to $650^{\circ} \mathrm{C}$, where the reflection suddenly drops. These two distinctly different thermal behaviors indicate two different grating types and can be classified into Type I and Type In behavior [7]. The grating that was written with $20 \mathrm{~kJ} / \mathrm{cm}^{2}$ shows a particularly weak reflection at room temperature. This grating can be assumed to inherit both grating types: Type I and Type In, which suppress each other. With an increasing temperature, the Type I grating anneals out and only the Type In grating becomes apparent. Hence, the grating reflection increases at around $400^{\circ} \mathrm{C}$. This grating can be seen as a transition from a Type I to Type In grating, which was previously believed to be a double regeneration [15]. Such transitions have been reported in the literature [11]. With respect to Fig. 3, this transition correlates to the dip in the GF4A reflection spectrum for different fluences. However, it is not apparent for SMF28, as this transition does not occur in hydrogen-loaded fiber. Figure 5 shows the results for the hydrogenloaded SMF28. The figure shows two fluences with the thermal behaviors of distinct grating types. The low fluence grating was written with $3 \mathrm{~kJ} / \mathrm{cm}^{2}$ and demonstrated a continuous grating reflection decay, which onsets at $100^{\circ} \mathrm{C}$. This grating

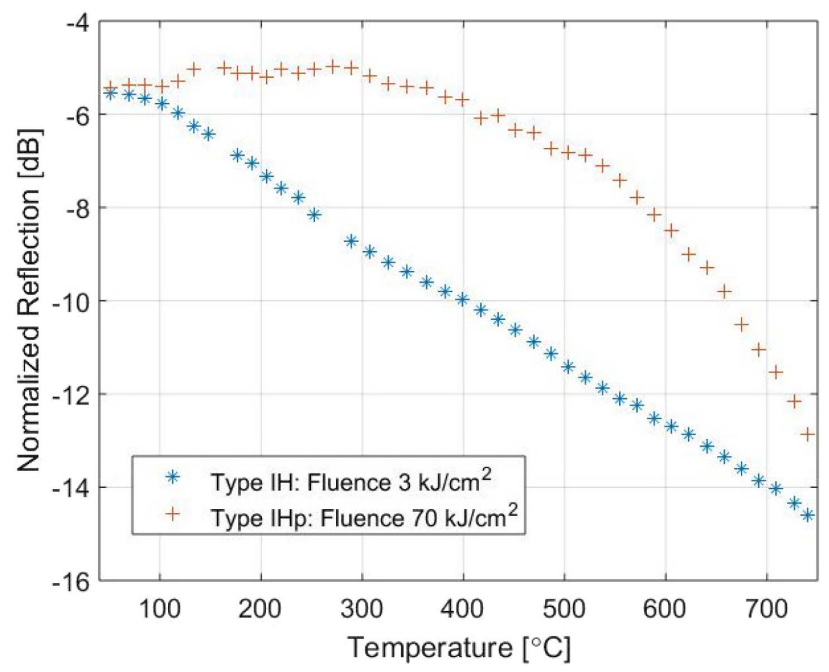

Fig. 5. Bragg reflection versus temperature for two distinct fluences in SMF28. The low fluence grating shows decreasing reflection at $100^{\circ} \mathrm{C}$, whereas the high fluence grating demonstrates thermal stability up to $400^{\circ} \mathrm{C}$.

demonstrates Type IH behavior, which has similar features to the Type I grating in non-hydrogen-loaded fibers. It has a lower thermal stability than the high fluence grating, which was written with $70 \mathrm{~kJ} / \mathrm{cm}^{2}$. This high fluence grating shows a thermal stability up to $400^{\circ} \mathrm{C}$. The higher fluence grating can be classified as Type IHp behavior, as this possesses significantly higher thermal stability.

In conclusion, this Letter identifies for the first time, to the best of our knowledge, the classification of grating types written with the SSDUW technique using thermal treatment. Depending on the energy that has been introduced to the fiber core during the writing process, a distinction between Type I and Type In in GF4A and Type IH and Type IHp in SMF28 has been accomplished. This method can be used specifically to characterize gratings defined through the sequential writing techniques, where the serial fabrication of the grating planes prohibits the definition of the grating type during the fabrication process. The classification is a destructive method, which reveals writing parameters linked to the grating type and, hence, can be used to write specific grating types in future runs. With these results, future FBGs written with the SSDUW system can be defined within the current framework and, hence, are comparable with FBGs written with other techniques.

Funding. Engineering and Physical Sciences Research Council (EPSRC) (EP/K034480/1, EP/M013243/1, EP/ M013294/1, EP/M024539/1, EP/M508147/1); Parker Aerospace, Parker Hannifin Corporation.

Acknowledgment. Data published in this Letter are available from the University of Southampton repository at http://dx.doi.org/10.5258/SOTON/D0669.

\section{REFERENCES}

1. S. J. Mihailov, Sensors 12, 1898 (2012).

2. J. Canning, Measurement 79, 236 (2016). 
3. R. Di Sante, Sensors 15, 18666 (2015).

4. A. L. Nosko, V. V. Mozalev, A. P. Nosko, A. V. Suvorov, and V. N. Lebedeva, J. Frict. Wear 33, 233 (2012).

5. X. Tomas Centrich, E. Shehab, P. Sydor, T. Mackley, P. John, and A. Harrison, Procedia CIRP 22, 287 (2014).

6. H. Xia, D. Byrd, S. Dekate, and B. Lee, J. Sensors 2013, 1 (2013).

7. J. Canning, Laser Photonics Rev. 2, 275 (2008).

8. D. P. Hand and P. St.J. Russell, Opt. Lett. 15, 102 (1990).

9. A. I. Gusarov and D. B. Doyle, Opt. Lett. 25, 872 (2000).

10. X. Shu, D. Zhao, L. Zhang, and I. Bennion, Appl. Opt. 43, 2006 (2004).
11. X. Shu, Y. Lui, D. Zhao, B. Gwandu, F. Florani, L. Zhang, and I. Bennion, Opt. Lett. 27, 701 (2002).

12. C. Sima, J. C. Gates, H. L. Rogers, P. L. Mennea, C. Holmes, M. N. Zervas, and P. G. R. Smith, Opt. Express 21, 15747 (2013).

13. M. Gagné, L. Bojor, R. Maciejko, and R. Kashyap, Opt. Express 16, 21550 (2008).

14. M. Gagné, S. Loranger, J. Lapointe, and R. Kashyap, Opt. Express 22, 387 (2014).

15. A. Jantzen, R. H. S. Bannerman, S. A. Berry, J. C. Gates, P. C. Gow, L. J. Boyd, P. G. R. Smith, and C. Holmes, Opt. Lett. 42, 3741 (2017). 